\title{
Retrain or Not Retrain? - Efficient Pruning Methods of Deep CNN Networks
}

\author{
Marcin Pietron ${ }^{(凶)}$ and Maciej Wielgosz \\ AGH - University of Science and Technology, Krakow, Poland \\ \{pietron,wielgosz\}@agh.edu.pl
}

\begin{abstract}
Nowadays, convolutional neural networks (CNN) play a major role in image processing tasks like image classification, object detection, semantic segmentation. Very often CNN networks have from several to hundred stacked layers with several megabytes of weights. One of the possible techniques to reduce complexity and memory footprint is pruning. Pruning is a process of removing weights which connect neurons from two adjacent layers in the network. The process of finding near optimal solution with specified and acceptable drop in accuracy can be more sophisticated when DL model has higher number of convolutional layers. In the paper few approaches based on retraining and no retraining are described and compared together.
\end{abstract}

Keywords: Deep learning $\cdot \mathrm{CNN} \cdot$ Pruning $\cdot$ Image processing

\section{Introduction}

The convolutional neural networks are the most popular and efficient model used in many AI tasks. They achieve best results in image classification, semantic segmentation, object detection etc. The reduction of memory capacity and complexity can make use of them in real-time applications like self driving cars, humanoid robots, drones etc. Therefore compression CNN models is a important step in adapting them in embedding systems and hardware accelerators. One of the methods to decrease memory footprint is a pruning process. In case of small convolutional network, the complexity of this process is much lower than in larger ones. In very deep CNN models which have several to few hundreds of convolutional layers the process of finding near global optimum solutions which guarantee acceptable drop in accuracy is quite a complex task. Genetic/memetic algorithms, reinforcement learning, random hill climbing or simulated annealing are good candidates to solve this problem. In paper, algorithm based on RMHC and simulated annealing methods is presented.

The pruning process can be done by two major methodologies. First one is a pruning a pre-trained networks, the second one is pruning using retraining. The first one is much faster. It needs only an inference step run on a test dataset in each stage/iteration of the algorithm, [2]. In case of mode with retraining 
pruning can be done after every weights updated in training process. This paper describes and compares the approaches using both the methodologies.

The squeezenet [9] model was one of the first approach in which compression by reducing the filters size was used. In this approach, architecture of alexnet was modified to create less complex model with same accuracy. Later approaches were concentrated more on quantization and pruning $[2,6]$ as a steps that enables compression. In [6] authors present approaches for CNN compression including pruning with retraining. The results for older architectures VGG and AlexNet are presented. In paper [8] authors describe reinforcement learning as a method for choosing channels for structural pruning. In article [7] the SNIP algorithm is described. The algorithm computes gradients during retraining and assigns priorities to weights based on gradients values. The pruning is done using knowledge about importance of weights in a training process. In papers $[4,5]$ compression for other machine learning models are described in NLP tasks. It is shown that by especially using sparse representations, it is possible to achieve better results than in baseline models. The paper is organized as follows. The Sect. 2 presents the methods for pruning pre-trained networks. There is a basic method and its further enhancements using more complex models analysis. The next Sect. 3 is about pruning with retraining on imagenet, CIFAR10 and CIFAR100 datasets and structural pruning. Finally, in Sect. 4 and 5 further work and conclusions are described.

\section{Pruning with No Retraining}

After the process of training neural model we acquire a set of weights for each trainable layer. These weights are not evenly distributed over the range of possible values for a selected data format. Majority of weights are concentrated around 0 or very close to it. Therefore, their impact on the resulting activation values is not significant. Depending on network implementation specifications, storing weights may require a significant amount of memory. Applying pruning process to remove some weights has a direct impact on lowering storage requirements. In this section the approaches based on pre-trained networks are presented. The first one is memetic approach which is based on random hill climbing with few extensions. The parameters to the heuristic were added to optimize and speed up the process of finding local optima solutions. In this algorithm pruning is a function that set weights values to zero whose magnitudes are below specific threshold (Eq. 1 and Eq. 2).

$$
\begin{gathered}
W_{p}=\text { pruning }(\text { threshold }, W) \\
W_{p}=\left\{\forall w_{i} \in W_{p}: w_{i}=0,\left|w_{i}\right|<\text { threshold } \vee w_{i}=w_{i} \in W\right\}
\end{gathered}
$$

Next, additionally more sophisticated analysis was incorporated to previous approach to improve obtained results. Presented method analyses energies/contributions of $2 \mathrm{D}$ filters in layers and heat maps to increase sparsity further. 


\subsection{Incremental Pruning Based on Random Hill Climbing}

The presented approach for fast pruning is based on random hill climbing and simulated annealing local search. In each iteration, it chooses specified number of layers to be pruned. The layers are chosen using probability distribution based on layers' complexities and sensitivities (Eq. 3, Eq.4, line 4). If a layer is more complex and less sensitive than others, it has more probability to be chosen. In each iteration, layers are pruned by the step which can be different and computed independently for each layer (line 7). If drop in accuracy is higher than given threshold reverse pruning is applied (the step can be cancelled or sparsities of different layers are decreased). Fitness function is a weighted sparsity which is overall memory capacity of current pruned model (line 11). Solution is a simple genotype where each layer is represented as a percentage of weights that were pruned in this layer. Algorithm can use as an option simulated annealing strategy which accepts worse solutions (exploration phase) to have the possibility to escape from local optima (line 18-22). In this case, in line 21 a next created solution can be worse than previous solution and will be accepted with specified probability which decreases in each iteration. Algorithm has a ranked list of all k-best solution already found (line 14). It helps to overcome algorithm stagnation by giving opportunity to return to good solutions (line 19). Each layer as it was mentioned earlier has sensitivity parameter which measures the latest impacts (number of impacts is defined by window size parameter) of this layer to the drop in accuracy of the model (Eq. 5, line 13). The layer sensitivity is updated after each iteration in which given layer is pruned (line 13). The step size which indicates percentage of weights to be pruned for a given layer is computed using current sensitivity value of a layer. If sensitivity is less than acceptable drop in accuracy (threshold) algorithm increases step size and vice versa using Eq. 6, line 24 .

$$
\begin{gathered}
\text { probability }_{i}=\text { size }_{i} \times\left(\text { threshold }- \text { sensitivity }_{i}\right) \\
\text { policy }=\text { categorical }(\text { probability }) \\
\text { sensitivity }_{i}^{t}=\frac{\sum_{t-\mid \text { window } \mid}^{t}(\text { baseline_acc }- \text { pruned_acc } t)}{\text { window }} \\
\text { step }_{i}^{t}=\text { step }_{i}^{t-1}+k \times \text { step }_{i}^{t-1} \times\left(\text { threshold }- \text { sensitivity }_{i}^{t-1}\right)
\end{gathered}
$$

The presented algorithm can be run in multi-layer mode in which, in one iteration more than one layer can be pruned. In Table 1 there are results achieved using Algorithm 1 with constant policy by running 150 iterations. Table contains weighted sparsities of pruned models and their drops from baseline accuracies. The threshold drop was set to 1.0. The Table 2 presents results using prioritization mode in which largest layers in given models were chosen for pruning in the first stage of the algorithm till the drop in accuracy is higher than given threshold. After that rest of the layers are pruned. We can observe significant 


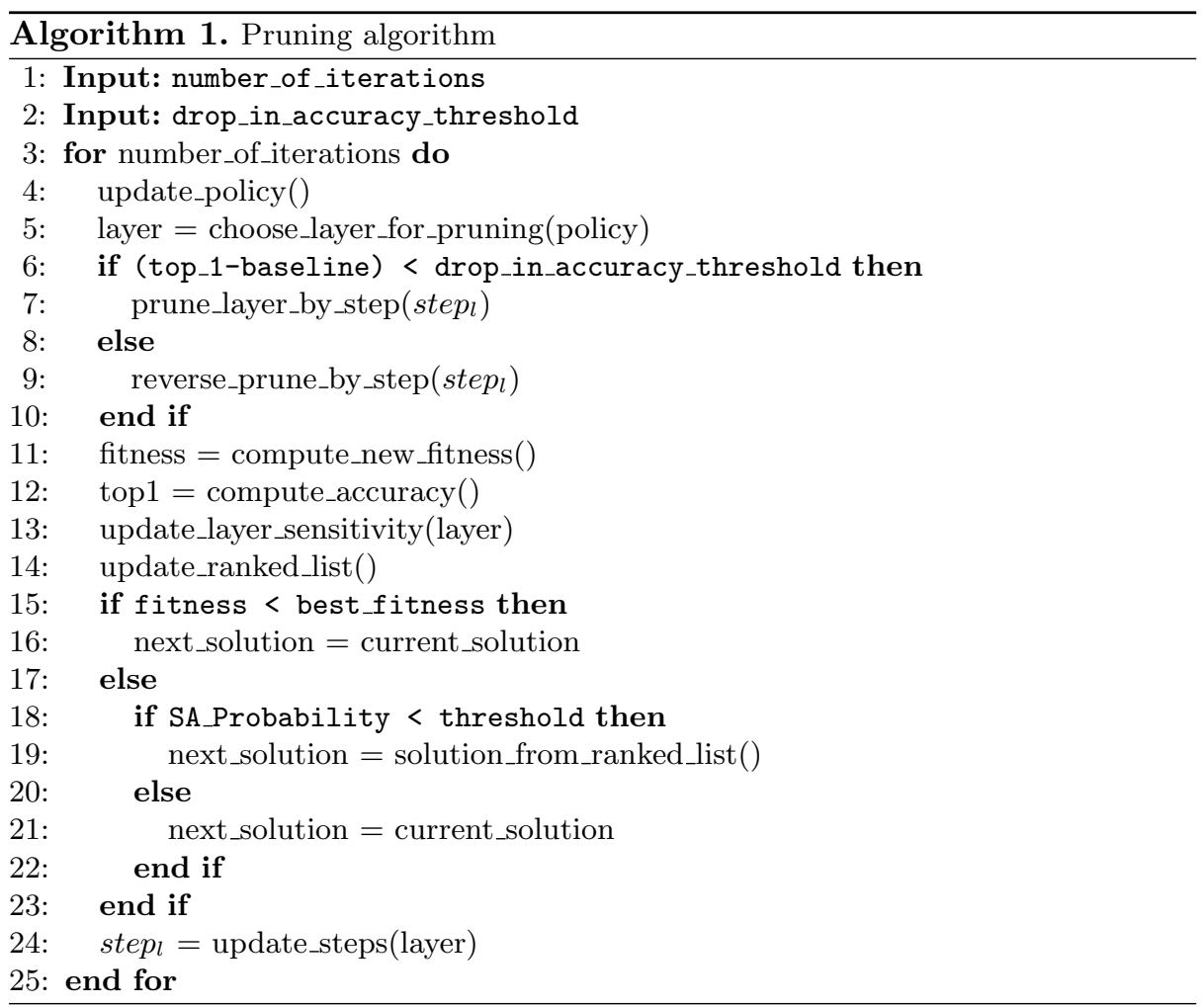

Table 1. Pruning results with constant policy

\begin{tabular}{l|l|l}
\hline Name & Weighted sparsity & $\mathrm{T} 1$ \\
\hline vgg16 & $35.3 \%$ & -0.8 \\
\hline resnet50 & $32.1 \%$ & -1 \\
\hline vgg19 & $32.6 \%$ & -1 \\
\hline inception_v3 & $18.1 \%$ & -0.8 \\
\hline
\end{tabular}

improvements in achieved results. Table 3 shows results of using dynamic policy updates during algorithm.

\subsection{D Filter and Its Activation Analysis for Further Pruning Improvements}

Improvement presented in this subsection does additional analysis that can explain the internal representation of the model and removes more weights with high probability to not decrease its accuracy. First approach is to compute 2D average filter contributions in a final answer of the network (Eq.7, Eq.8, Eq. 9). The next one is to analyze filter contribution in a process of recognition 
specific class. Each class is analyzed separately and average neurons activations are measured. Then in each layer we can extract region of weights that are less important in the whole process of recognition using some threshold of importance. In Table 4 and Table 5 there are results presented for these two steps performed on the last layer before softmax in VGG16 after running Algorithm 1. It shows that is possible to do further pruning to increase sparsity without drop in the accuracy.

$$
F=\left\{f_{m n h w} \in R: m \in M, n \in N, h \in H, w \in W\right\}
$$

where: M, N, H, W are number of channels, kernels, height and width respectively of layer filter

$$
\begin{aligned}
F \_2 D & =\left\{f_{m n} \in R: \in R: m \in M, n \in N\right\} \\
f \_2 D \_ \text {_energy } i j & =\text { mean }\left(\text { sum }\left(\left(f_{\_} 2 D_{i j}\right) \odot \text { receptive_field }{ }_{i j}\right)\right)
\end{aligned}
$$

Table 2. Pruning results with specified prioritization

\begin{tabular}{l|l|l|l}
\hline Name & Weighted sparsity & T1 drop & Prioritization list \\
\hline vgg16 & $67 \%$ & -0.9 & Layers 14, 15, 16 \\
\hline resnet50 & $37 \%$ & -1.1 & 5 largest layers \\
\hline vgg19 & 65 & -1.0 & Layers 17, 18, 19 \\
\hline inception_v3 25 & $35 \%$ & -0.9 & 8 largest layers \\
\hline
\end{tabular}

Table 3. Results of pruning with dynamic policy

\begin{tabular}{l|l|l}
\hline Name & Weighted sparsity & T1 drop \\
\hline $\operatorname{vgg} 16$ & $65 \%$ & -1.0 \\
\hline resnet50 & $35 \%$ & -1.0 \\
\hline vgg19 & $60 \%$ & -0.9 \\
\hline inception_v3 & $24 \%$ & -0.9 \\
\hline
\end{tabular}

Table 4. VGG16 with 2D filter analysis

\begin{tabular}{l|l|l|l|l}
\hline Name & T1 & Pruning & With 2D & T1 drop \\
\hline CIFAR10 & $90.76 \%$ & $50 \%$ & $52 \%$ & -1.0 \\
\hline CIFAR100 & $77.6 \%$ & $45 \%$ & $47 \%$ & -1.0 \\
\hline
\end{tabular}

Table 5. VGG16 with 2D filter analysis and filter contributions in a classes recognition

\begin{tabular}{l|l|l|l|l}
\hline Name & T1 & Pruning & +Filter contributions & T1 drop \\
\hline CIFAR10 & $90.55 \%$ & $50 \%$ & $54 \%$ & -1.0 \\
\hline CIFAR100 & $77.5 \%$ & $45 \%$ & $50 \%$ & -1.0 \\
\hline
\end{tabular}




\section{Pruning with Retraining}

The methods described in the previous section have one main drawback, their weight can be fine tuned during the pruning process to boost model accuracy. The training step can improve accuracy of pruned network by learning weights that were not removed before. In this section, results of these methods are presented.

\subsection{Methods}

Retraining is recognized as an effective method for regaining performance of the pruned model. However, it is important to pick a right protocol and retraining parameters. We have examined three different schemes of pruning and retraining.

- simple retraining which without masking,

- simple retraining with masking,

- adaptive retraining with boosting.

The first two methods apply a simple retraining procedure after each step of pruning. The procedure can be interleaved with masking operation. It is implemented by zeroing gradient which otherwise would be applied to the pruned weights. It is worth noting that even without masking the pruned weights are mode prone to be pruned again in the next epoch because they are small. Consequently, the masking operation makes the pruning process more stable since a pool of pruned weights is progressively enlarged without change of coefficients. The simple method is limited in its effectiveness mostly because it lacks ability to adopt pruning both in terms of layers of the model and the retraining time. Some layers during selected training epochs are more prone to pruning, which is not taken into account in the simple method. Therefore, we have proposed the retraining with boosting procedure which is given by Algorithm 2 .

The proposed approach Algorithm 2 relies on a choice of priority list of the layers which is supposed to be set at the very beginning of the process. The rest of the parameters steps decide how many steps are taken before scale is changes. This gradually reduces pruning factor. The scale (refer to Algorithm 2) decides how many time the step is reduced. Once the model is pruned it is validated with a small dataset to check if the performance drop is not to large. If this is the case the process of pruning is stopped for the given layer in this iteration (epoch) and the algorithm goes to the next layer on the priority list. The pruning process may terminate in a regular fashion when all the steps and scale rates are exhausted. In order to speedup the process a layer which was skipped several times due to the performance drop after pruning is marked as permanently skipped. It is worth noting that a number of epochs should be picked properly in order to satisfy the number of the protocol interactions (number of steps and scale changes). 


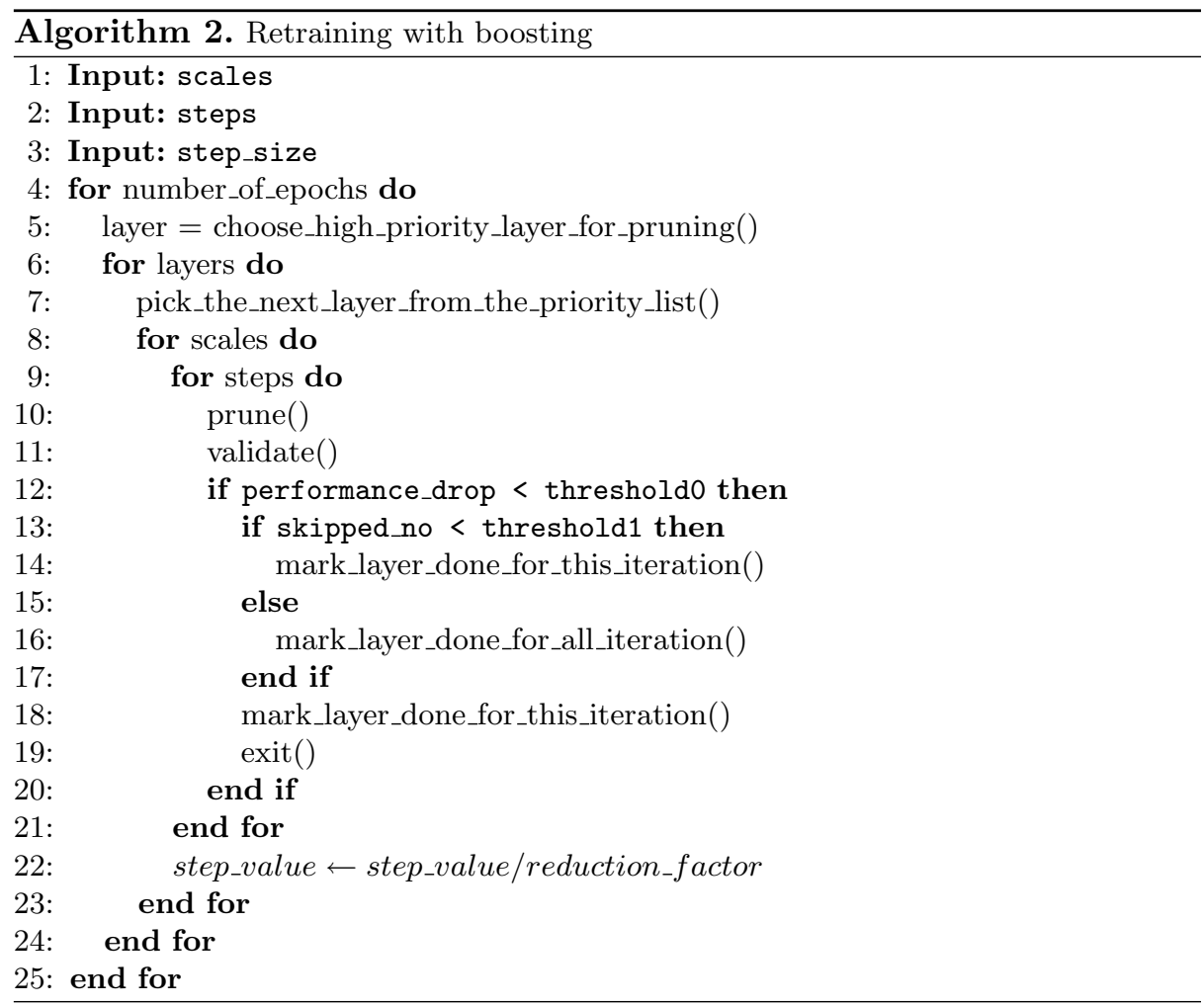

\subsection{Results of the Pruning and Retraining Experiments on Imagenet}

Table 6. Results of Resnet-50 simple pruning and retraining

\begin{tabular}{l|l|l|l|l|l|l|l}
\hline Layers pruned & Sparsity & Masking & Best T1 & T1 drop & Best T5 & Epoch & lr (reduction) \\
\hline None & 0 & None & $76.13 \%$ & 0 & $92.862 \%$ & 103 & $0.1(30)$ \\
\hline all-0.2 & All layers 0.2 & False & $76.83 \%$ & 0.7 & $93.15 \%$ & 14 & $1.00 \mathrm{E}-03$ \\
\hline all-0.3 & All layers 0.3 & False & $76.95 \%$ & 0.82 & $93.21 \%$ & 68 & $1.00 \mathrm{E}-03$ \\
\hline all-0.7 & All layers 0.7 & True & $59.55 \%$ & -16.58 & $83.62 \%$ & 26 & 0.1 \\
\hline
\end{tabular}

Table 7. Results of Resnet-50 progressive pruning and retraining

\begin{tabular}{l|l|l|l|l|l|r|l}
\hline Layers pruned & Sparsity & Masking & Best T1 & T1 drop & Best T5 & Epoch & lr (reduction) \\
\hline None & 0 & None & $76.13 \%$ & 0 & $92.862 \%$ & 103 & $0.1(30)$ \\
\hline all-0.2+0.1* epoch & all layers 0.3 & False & $76.48 \%$ & 0.35 & $93.07 \%$ & 1 & $1.00 \mathrm{E}-03$ \\
\hline all-0.1+0.1* epoch & all layers 0.2 & True & $76.56 \%$ & 0.43 & $93.1 \%$ & 1 & $1.00 \mathrm{E}-03$ \\
\hline all-0.1+0.01* epoch & all_layers_0.53 & True & $76.09 \%$ & -0.04 & $93 \%$ & 43 & $0.01(30)$ \\
\hline
\end{tabular}


There was series of experiments conducted as presented in Table 6,7 and 8 . Different parameters were chosen as well as different strategies were tested. In the first a naive approach was explored as a baseline. The results are presented in Table 6. We can see that equal pruning of all the layers for 0.2 and 0.3 sparsity led to the boost of the model performance. However, more aggressive pruning of 0.7 equal sparsity resulted in a significant decline of the sparsity. The proposed simple method may be useful when treated as a form of regularization and slight increase of the model sparsity.

Table 8. Results of Resnet-50 boosted pruning and retraining

\begin{tabular}{l|l|l|l|l|l|l|l}
\hline Layers pruned & Sparsity & Masking & Best T1 & T1 drop & Best T5 & Epoch & lr (reduction) \\
\hline None & 0 & None & $76.13 \%$ & 0 & $92.862 \%$ & 103 & $0.1(30)$ \\
\hline Steps: 12, scales: 2, step: 0.05 & Weighted: 0.37 & True & $75.12 \%$ & -1.01 & $92.57 \%$ & 47 & $1.00 \mathrm{E}-03$ \\
\hline Steps: 12, scales: 2, step: 0.05 & Weighted: 0.42 & True & $75 \%$ & -1.13 & $93.09 \%$ & 30 & $1.00 \mathrm{E}-03$ \\
\hline Steps: 12, scales: 2, step: 0.05 & Weighted: 0.427 & True & $79.88 \%$ & 3.75 & $94.96 \%$ & 100 & $1.00 \mathrm{E}-03$ \\
\hline Steps: 12, scales: 2, step: 0.05 & Weighted: 0.57 & True & $75.35 \%$ & -0.78 & $92.59 \%$ & 299 & $1.00 \mathrm{E}-03$ \\
\hline Steps: 4, scales: 2, step: 0.05 & Weighted: 0.5137 & True & $75.61 \%$ & -0.52 & $92.67 \%$ & 431 & $1.00 \mathrm{E}-03$ \\
\hline Steps: 6, scales: 2, step: 0.05 & Global: 0.57 & True & $75.52 \%$ & -0.61 & $92.68 \%$ & 279 & $1.00 \mathrm{E}-03$ \\
\hline Steps: 10, scales: 2, step: 0.05 & Global: 0.44 & True & $76.23 \%$ & 0.1 & $93 \%$ & 141 & $1.00 \mathrm{E}-03$ \\
\hline Steps: 2, scale: 4, step: 0.2 & Global: 0.648 & True & $74.51 \%$ & -1.62 & $92.19 \%$ & 88 & $1.00 \mathrm{E}-03$ \\
\hline
\end{tabular}

It is worth noting that progressive pruning which results are presented in Table 7 is much more effective. For instance, the experiment with starting point of 0.1 and progress of 0.01 every epoch (see the last row in Table 7 ) allowed to reach equal sparsity of $53 \%$ after 43 epochs with negligible loss of performance. This method despite its benefits is limited in its capacity to reduce sparsity. Method saturates at about $60 \%$ of sparsity. The most advanced approach of pruning and retraining in the boosting method given by Algorithm 2. Its results are presented in Table 8 . We can in Table 8 that different values of steps and scales lead to huge discrepancies in the results in terms of sparsity. The highest sparsity of $64.8 \%$ was achieved for steps: 2 , scale: 4 and step value: 0.2 . This was achieved at the expanse of noticeable loss of the performance. On the other hand small step value, large number of steps and training epochs lead to much lower performance degradation as proved by the experiment with steps: 6 , scales: 2, step value: 0.05 and 279 epochs of training. However, such large number of epochs required approx. 10 days of training time on 8 Nvidia GTX 1080 GPUs.

Choice of a proper number of steps, scales and step values should be done individually for each model and ideally facilitated with an optimization algorithm.

During a pruning and retraining operation of a pretrained model with high learning rate, there is a huge degradation of the performance ( $t 1$ and $t 5)$ in the very first epoch as presented in Fig. 1. In the next epochs the model regains it original performance quite fast. The presented in Fig. 1 resembles in terms of a training pattern most of the experiments showed in Table 8. 


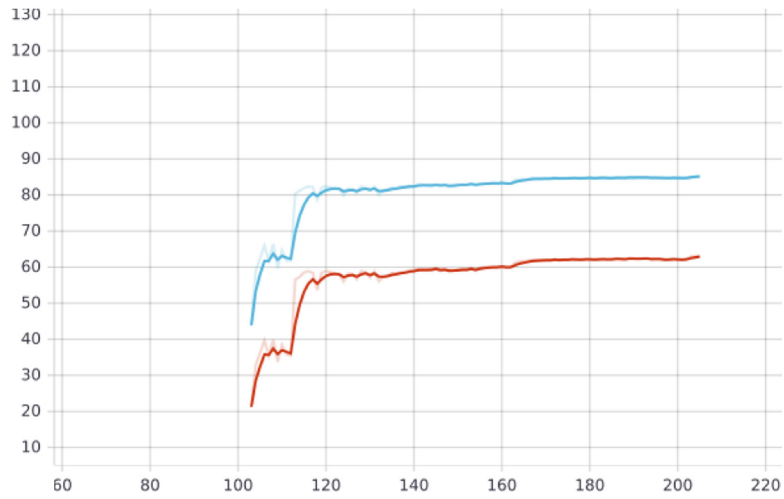

Fig. 1. Retraining of the pretrained Resnet50 with global sparsity of $20 \%$. Retraining starts at 104 epoch. Top5 is marked in blue and Top1 in red. (Color figure online)

\subsection{Pruning with Retraining on CIFAR Datasets}

The similar approach as described in the previous section was performed on a CIFAR10 and CIFAR100 datasets. The main difference is that in each step, the weights for pruning were chosen using its gradient values. This information gives feedback how important the weight was in the former training step (Algorithm 3). If its significance is less then it is safer to remove it. Table 9 and Table 10 present results obtained using Algorithm 3. They show significant improvement in the sparsity obtained when compared to fast pruning approach.

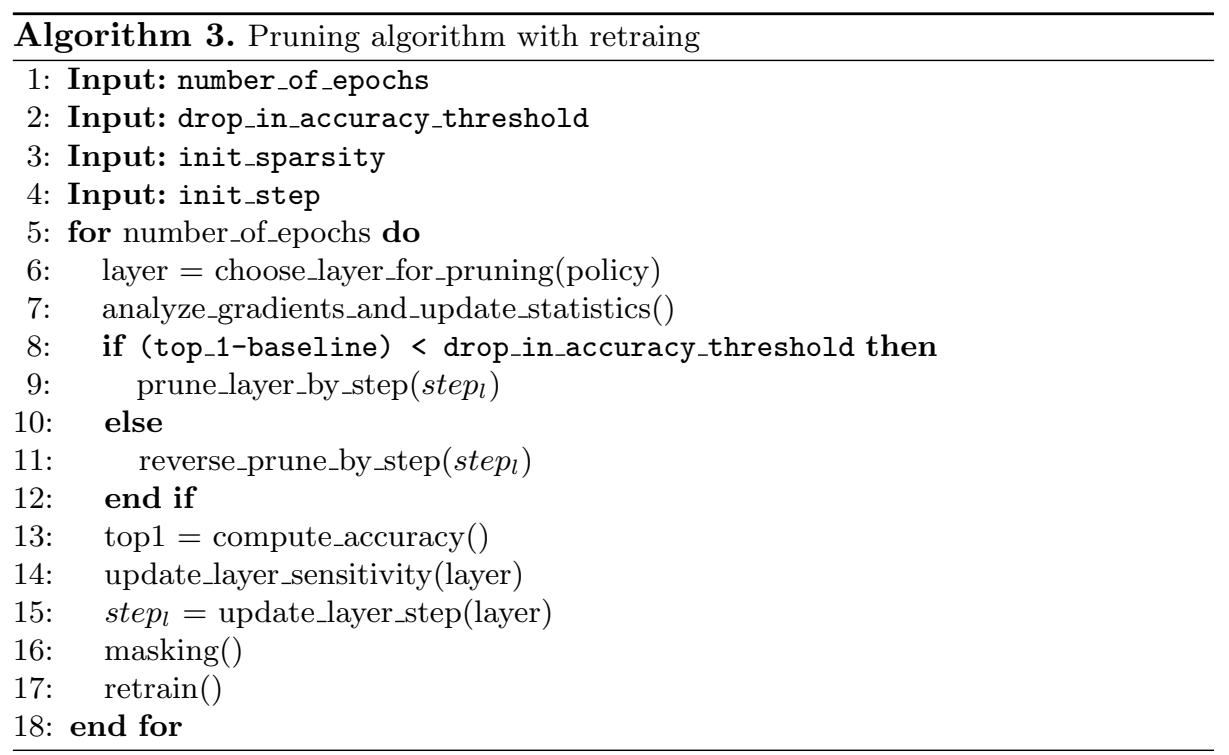


Table 9. Results of fine-grain pruning with retraining (CIFAR10)

\begin{tabular}{l|l|l|l}
\hline Name & Baseline T1 & Pruned T1 & Pruned size \\
\hline vgg19 & $92.37 \%$ & $91.8 \% 1$ & $2 \%$ \\
\hline resnet50 & $95.26 \%$ & $94.99 \%$ & $8 \%$ \\
\hline
\end{tabular}

Table 10. Results of fine-grain pruning with retraining (CIFAR100)

\begin{tabular}{l|l|l|c}
\hline Name & Baseline T1 & Pruned T1 & Pruned size \\
\hline vgg19 & $70.62 \%$ & $70.12 \%$ & $5 \%$ \\
\hline resnet50 & $78.21 \%$ & $77.56 \%$ & $22 \%$ \\
\hline
\end{tabular}

\subsection{Structural Pruning}

Structural pruning is a process where blocks of weights are removed. One of the most popular is reducing number of channels in a filter. Using this approach, straightforward implementation on many hardware accelerators can speed up original network without any software modification. Reducing the number of channels (chunk of weights) in a pretrained network usually affects, significantly, model accuracy. This approach should be mixed with training steps to minimize the accuracy drop. In the presented approach, the channels with lowest L1 norm and lowest variance among $2 \mathrm{D}$ filters inside given channel were chosen to be removed. The subset of such channels were extracted in each iteration. Then retraining process was run to increase accuracy. The process was performed till drop in accuracy was higher than given threshold (1\%). The results are presented in Table 11, Table 12. It is worth noting that results achieved using this approach are significantly worse than in fine grain pruning and the process is significantly slower than presented fast pruning algorithm.

Table 11. Results of structural pruning with retraining (CIFAR10)

\begin{tabular}{l|l|l|l}
\hline Name & Baseline T1 & Pruned T1 & Pruned size \\
\hline vgg19 & $92.37 \%$ & $92.42 \%$ & $52 \%$ \\
\hline resnet50 & $95.26 \%$ & $94.98 \%$ & $72 \%$ \\
\hline
\end{tabular}

Table 12. Results of structural pruning with retraining (CIFAR100)

\begin{tabular}{l|l|l|l}
\hline Name & Baseline T1 & Pruned T1 & Pruned size \\
\hline vgg19 & $70.62 \%$ & $70.71 \%$ & $54 \%$ \\
\hline resnet50 & $78.21 \%$ & $77.50 \%$ & $78 \%$ \\
\hline
\end{tabular}




\section{Conclusions}

The results presented in this paper show quite high disparities in sparsities between pruning with retraining or without retraining. Retraining can significantly improve the drop in accuracy after pruning. During retraining process, other aspects like masking, step size of the pruning at a current stage of pruning process are very important to achieve better results. The same effect can be observed in fast pruning on pretrained networks. It is worth noting about the time difference between these two pruning approaches. In case of pruning without retraining, it is possible to prune the very deep networks from several minutes to $2-3 \mathrm{~h}$. The time depends on the size of testing the dataset. In case of using retraining, many epochs should be run to achieve satisfactory level of sparsity with a very small drop in accuracy. In case of Imagenet, one epoch lasts for approximately one hour. The overall process takes a few days. Choosing the method depends on hardware accelerator which will be used after pruning. If given hardware can make use of lower sparsity then pruning without retraining can be fast and efficient. In case of accelerator, it needs very high sparsity, slow pruning with retraining should be performed. The last conclusion is that structure pruning without retraining doesn't guarantee low drop in accuracy. It should be run with retraining.

\section{Further Work}

Further work will concentrate on tuning hyper-parameters in pruning algorithms which were described in a paper. It is still an open question if it is possible or how to find common rules for pruning all CNN networks to achieve satisfactory result. The next issue to focus on will be speeding up the pruning with retraining process by using more knowledge and statistics about the network. The proposed pruning methods of Deep Learning architectures can also be optimized and tested on a system level by taking data into consideration. This can be pronounced especially in latency critical systems [10].

\section{References}

1. Pietron, M., Karwatowski, M., Wielgosz, M., Duda, J.: Fast compression and optimization of deep learning models for natural language processing. In: Proceedings of the CANDAR 2019, Nagasaki. IEEE Explore (2019)

2. Al-Hami, M., Pietron, M., Casas, R., Wielgosz, M.: Methodologies of compressing a stable performance convolutional neural networks in image classification. Neural Process. Lett. 51(1), 105-127 (2019). https://doi.org/10.1007/s11063-019-10076-y

3. Al-Hami, M., Pietron, M., Casas, R., Hijazi, S., Kaul, P.: Towards a stable quantized convolutional neural networks: an embedded perspective. In: 10th International Conference on Agents and Artificial Intelligence (ICAART), vol. 2, pp. 573-580 (2018) 
4. Wróbel, K., Wielgosz, M., Pietroń, M., Karwatowski, M., Duda, J., SmywińskiPohl, A.: Improving text classification with vectors of reduced precision. In: Proceedings of the ICAART 2018: 10th International Conference on Agents and Artificial Intelligence, vol. 2, pp. 531-538 (2018)

5. Wróbel, K., Pietroń, M., Wielgosz, M., Karwatowski, M., Wiatr, K.: Convolutional neural network compression for natural language processing. arXiv preprint arXiv:1805.10796 (2018)

6. Han, S., Mao, H., Dally, W.J.: Deep compression: compressing deep neural networks with pruning, trained quantization and Huffman coding. In: ICLR 2016. arXiv preprint arXiv: 1510.00149

7. Lee, N., Ajanthan, T., Torr, P.H.S.: SNIP: single-shot network pruning based on connection sensitivity. In: ICLR 2019. arXiv preprint arXiv:1810.02340 (2018)

8. Huang, Q., Zhou, K., You, S., Neumann, U.: Learning to prune filters in convolutional neural networks. arXiv preprint arXiv:1801.07365 (2018)

9. SqueezeNet. arXiv preprint arXiv:1804.09028 (2018)

10. Wielgosz, M., Marutiz, P., Jiang, W., Rønningen, L.A.: An FPGA-baed platform for a network architecture with delay guarantee. J. Circuits Syst. Comput. 22(06) (2013). https://doi.org/10.1142/S021812661350045X 